The mean $( \pm \mathrm{SD})$ age was $39.1 \pm 12.6$ years. Ophthalmological examination was abnormal in 51 patients $(71.8 \%)$ and normal in 20 patients $(28.2 \%)$. Ocular complaint was recorded in $33.8 \%$ of patients. The primary lesion in the anterior segment was conjunctivitis (31\%). The most common lesions of the posterior segment were cytomegalovirus retinitis (7.1\%) and HIV retinopathy $(5.7 \%)$. The mean CD4 count in our study was $206.8 \pm 229.8$ cells $/ \mathrm{mm}^{3}$.

$71.4 \%$ of patients with ocular manifestations had a CD4 count less than 200 cells $/ \mathrm{mm}^{3}$.

Ocular manifestations and CD4 cells count, no significant statistical relationship was found $(\mathrm{P}=1,0000)$.

Conclusion With access to antiretroviral therapy, ocular manifestations of HIV/AIDS are increasingly less frequent. Ocular manifestations were not related to the CD4 cells count in this study.

This study, the first in our area, will guide the ophthalmologist and let him play its role mainly in the diagnosis and care of patients.

\section{P2.124 DERMATOLOGICAL CONDITIONS IN HIV INFECTED PATIENTS IN TURKEY}

doi:10.1136/sextrans-2013-051184.0388

II Ertam, 'S Yıldı, ${ }^{2} \mathbf{D}$ Gökengin. 'Ege University, Fac of Medicine, Dept of Dermatology and Venereology, Izmir, Turkey; ${ }^{2}$ Ege University, Fac of Medicine, Dept of Infectious Diseases and Clinical Microbiology, Izmir, Turkey

Background Dermatological conditions are common manifestations in HIV infected patients. They may appear as the initial presentation of HIV infection, during the course of the infection or as adverse events due to antiretroviral treatment. The aim of this study was to determine the prevalence of skin diseases in HIV infected patients monitored in an infectious diseases clinic in Turkey.

Methods This study included HIV positive patients monitored in the Department of Infectious Diseases and Clinical Microbiology (IDCM), Faculty of Medicine, Ege University, Izmir, Turkey. According to a protocole between the Department IDCM and Department of Dermatology and Venereology, starting from February 2011, all patients presenting to the IDCM clinic for the first time with an HIV infection were screened for dermatological conditions on first visit and on each following visit by the infectious diseases physician. In addition, patients who have been monitored in the same clinic were questioned for any dermatological complaints on each visit. Patients with a dermatological condition were referred to the Dermatology and Venereology Clinic for further diagnosis and treatment. The records of the patients were reviewed retrospectively.

Results The study comprised 187 HIV-infected patients from February 2011 to February 2013. Twenty-four patients (12.8\%) were diagnosed with 40 dermatological conditions. While 16 patients $(66.6 \%)$ had only one dermatological condition, 8 patients had more than one condition ( 3 patients with 2 dermatological manifestations, 2 patients with 3, 2 patients with 4 and 1 patient with 5). The most common dermatological manifestations were herpes genitalis (3 patients) and genital warts ( 3 patients), followed by molluscum contagiosum (2 patients).

Conclusion Through screening of HIV-infected patients for skin manifestations and collaboration with the Dermatology and Venereology Clinic may result with earlier diagnosis and treatment of the skin disease and a higher quality of life for the patient.

\section{P2.125 TEMPORAL ORDER OF INFECTION WITH HPV AND C. TRACHOMATIS INFECTION IN THE DEVELOPMENT HIGH- GRADE CIN}

doi:10.1136/sextrans-2013-051184.0389

J Paavonen. Department of Obstetrics and Gynecology, University Hospital, Helsinki, Finland
Interaction between HPV and C. trachomatis $(C T)$ in cervical carcinogenesis is not well understood. We studied the joint effects of HPV and CT on the risk of developing high-grade CIN or adenocarcinoma in situ (AIS). In a cohort of 94,349 women with prediagnostic paired serum samples, 490 women developed cervical CIN3/AIS. Serum IgG antibodies to HPV6/16/18/31/33/45 and CT were measured by ELISA. Control group consisted of a subcohort of 2976 women without CIN3/AIS. The cutoff antibody levels for different HPV types were predefined. CT antibody level was defined as negative or positive. Rate ratios (RR) and variances were estimated by Cox model. Since we were interested in the risk associated with the temporal order of infections, the reference group was dual seropositivity at the first serum sampling. When comparing the temporal exposure, we noted high RR of CIN3/AIS for incident CT and hrHPV clade A7 (HPV18/45) infection (RR 25, 95\% CI 3.8, 170) compared to the reference group. Concomitant incident infection with HPV18 and CT or HPV45 and CT was associated with very high risk for CIN3/AIS. Adjustment for HPV16 had no effect on the point estimates. The high risk associated with incident (within on average 2.4 years) CT and HPV18/45 infections is in line with the HPV16- independent role of CT in the development of CIN3/ICC shown before. In conclusion, the temporal order of infections has an effect on the development of CIN3/AIS. The risk associated with concomitant CT and HPV18/45 infection suggests that HPV vaccination and screening for hrHPV and CT play significant roles in cervical cancer prevention.

\section{P2.126 KAPOSI'S SARCOMA PATIENT PROFILE IN THE COHORT OF THE GERMAN COMPETENCE NETZWORK HIV/AIDS}

doi:10.1136/sextrans-2013-051184.0390

'R E Scheitz, ${ }^{2,3}$ S Esser, ${ }^{1,3,4} \mathrm{~N}$ H Brockmeyer, ${ }^{5,3} \mathrm{~A}$ Moll, ${ }^{6,3}$ S Dupke, ${ }^{7,3} \mathrm{M}$ Rausch, ${ }^{8,3} \mathrm{H}$ Rasokat, ${ }^{9,3} \mathrm{C}$ Michalik, ${ }^{10,3} \mathrm{~S}$ Scholten, ${ }^{1,3} \mathrm{~A}$ V Potthoff. ${ }^{1}$ Clinic for Dermatology, Venerology and Allergology, Ruhr-Universität Bochum, Bochum, Germany; ${ }^{2}$ Clinic for Dermatology and Venerology, University Clinic Essen, Essen, Germany; ${ }^{3}$ Competence Network for HIV/ AIDS, Bochum, Germany; ${ }^{4}$ German STI-Society (DSTIG), Bochum, Germany; ${ }^{5}$ Practice Center Kaiserdamm, Berlin, Germany; ${ }^{6}$ Private practise Driesener Strasse, Berlin, Germany; 'Doctors Center Nollendorfplatz, Berlin, Germany; ${ }^{8}$ Clinic and Polyclinic for Dermatology and Venerology, University Clinic Cologne, Cologne, Germany; ${ }^{9} \mathrm{Centre}$ for Clinical Trials, Cologne, Germany; ${ }^{10}$ Private practise Hohenstaufenring, Cologne, Germany

Background The aim of this study is the acquisition and description of patients with HIV-associated Kaposi-sarcoma (KS) from the cohort database of the competence network for HIV/AIDS.

Patients and Methods Data from patients with diagnosis of KS from 9 centres of the competence network for HIV/AIDS were evaluated. The baseline characteristics at the time of the initial KS diagnosis were described. Recurrence and survival probabilities were estimated by means of Kaplan-Meier curves. The influence factors for survival were analysed with multiple Cox regression models. As potential risk factors for the mortality risk the CD4- and CD8 cell count, HIV-viral load value, antiretroviral therapy (ART), chemotherapy and the patient's age were evaluated.

Results In the period of 1987-2011 KS was diagnosed in 222 study participants, $97.7 \%$ were men and $2.3 \%$ women. The most common HIV-transmission risk was MSM (88.3\%). The median age was 38.5 years (min. 23.4; max. 76.8). 70.5\% of the patients showed exclusively cutaneus presentation. The median value of HIV viral load was 37,000 copies per $\mathrm{ml}$ at the initial diagnosis of $\mathrm{KS} .55 .5 \%$ of the patients had a CD4 cell count under 200/ $\mu$ l. A KS therapy was documented for 198 patients. The 222 study participants were clustered according their therapies; ART only and ART in combination with other treatments, e.g. liposomal doxorubicin or other chemotherapeutic regimen, local excision, radiation and/or interferon-alpha. It was shown that about $80 \%$ of the patients had no recurrence after the therapies and 18 patients died. The 5 and 10-year survival 
probabilities were $96.8 \%$ and $91.3 \%$. In addition it was shown by the Cox-model that patients with higher CD4 and patients without ART before KS diagnosis had a significant lower mortality risk.

Conclusion A low CD4 cell count at KS diagnosis is an important predictor for mortality.

\section{P2.127 PRESENTATION OF LYMPHOGRANULOMA VENEREUM (LGV) IN 2012 IN THE REFERENCE STI UNIT OF BARCELONA}

doi:10.1136/sextrans-2013-051184.0391

'M Arando, 'M Vall, 'P Armengol, ${ }^{2} \mathrm{E}$ Caballero, 'M Barberá. 'UITS, Barcelona, Spain; ${ }^{2}$ Hospital Universitari Vall d'Hebron, Barcelona, Spain

Since 2003 LGV outbreaks have been reported in different European cities in men who have sex patient men with high-risk sexual behaviours. In the majority of cases the most common clinical presentation was a rectal syndrome described as an acute proctitis. In Barcelona during 2012 the most common clinical presentation was proctitis, but revealed an increase in cases of LGV extrarectal location.

Objective To analyse LGV cases that have been diagnosed in 2012 in the reference STI Unit (UITS) Drassanes of Barcelona.

Methods Retrospective descriptive study. Review of epidemiological data, clinical presentation, physical examination and results of mentioned cases has been conducted.

Results In 2012 fifty-two cases of LGV in UITS were diagnosed, 8 of them in an extrarectal location. All of them were male: 50 were MSM and 2 bisexual men. Co-infection with HIV was $65 \%$, decreasing to $50 \%$ in the extrarectal location. $11.5 \%$ of them had a history of previous LGV and $36.5 \%$ had a sexually transmitted infection in the previous 12 months. The most common clinical presentation was proctitis $(65 \%)$, other clinical manifestations were urethritis, inguinal syndrome and anogenital ulcers. $21 \%$ were diagnosed by contact study (half for LGV and half for other STI) and one case was diagnosed by screening in an asymptomatic patient.

Discussion Although during the last years the typical presentation of LGV has been proctitis, the extrarectal location has increased considerably in 2012. In this situation it is necessary to assess whether or not to request it in a urethral syndrome as ulcerative lesions, especially in men who have sex with men with high-risk sexual behaviours.

\section{P2.128 GENITAL PRIMARY LYMPHOGRANULOMA VENEREUM}

doi:10.1136/sextrans-2013-051184.0392

M Vall-Mayans, E Caballero. University Hospital Vall d'Hebron, Barcelona, Spain

Lymphogranuloma venereum (LGV) in Europe emerged in 2003 affecting mostly MSM. Rectal primary LGV causing proctitis has been by far de most common clinical manifestation. In Barcelona, with a cumulative number of around 200 cases up to 2012, some cases have appeared with extrarectal manifestations since 2008. We report two cases in MSM with genital primary LGV mimicking primary syphilis.

Case 1. A 29-year-old HIV-positive man presented with a genital chancre and inguinal lymphadenopathy of 10 days' duration. He reported 5 casual partners the last 3 months. Although dark-field exam was negative the patient was treated with benzathine penicillin G 2.4 million units. He was seen 4 days later without improvement, with buboes and genital edoema. A swab from the ulcer was positive for LGV and negative for syphilis and chancroid by PCR. A diagnosis of bubonulus was made, and the patient started doxycycline $100 \mathrm{mg} / 12 \mathrm{~h}$ for 21 days. He improved persisting a small residual soft mass on the shaft of the penis.

Case 2. A 37-year-old HIV-negative man presented with a genital ulcer and lymphadenopathy of 2 days' duration. He had multiple unprotected casual partners in sex parties 3 days before. Three consecutive dark-field exams were negative. Five days later the ulcer persisted like a chancre and inguinal buboes were more evident. LGV was detected from the ulcer while syphilis and herpes were negative by PCR. The clinical manifestations resolved quickly after completion of treatment with doxycycline as above.

Cases with non-rectal lesions would be expected as LGV spreads. When present, the most common presentation of the primary lesion in the genitalia is a nonindurated herpetiform evanescent ulcer. Clinicians must be vigilant to the evolving faces of LGV, be prepared to recognise the full manifestations of this disease and consider LGV in the differential diagnosis of genital chancre.

\section{P2.129 PREVALENCE OF MALARIA IN HIV PATIENTS UNDER COTRIMOXAZOLE, CASE OF KINDU, MANIEMA, D.R.CONGO}

doi:10.1136/sextrans-2013-051184.0393

'A M Bulabula, ${ }^{2} \mathrm{~N}$ A Bulabula, ${ }^{3} \mathrm{M}$ Mbo, ${ }^{3} \mathrm{~T}$ C Kayembe. ${ }^{1}$ Universite De Kindu, Kindu, Congo; 2 Provincial General Hospital of Bukavu, Bukavu, Congo; ${ }^{3}$ Universite de Kisangani, Kisangani, Congo

Background Malaria and HIV/AIDS are common in sub-Saharan Africa, their association aggravates the evolution of one another condition. HIV patients under cotrimoxazole have been reported to present less malaria cases. This study aims to determine the prevalence of malaria among HIV patients under Cotrimoxazole in Kindu.

Methods A sample of 1533 subjects, including 511 people living with HIV (PLHIV) attending the Voluntary Testing Centre (VCT) of the General Hospital of Kindu and 1022 controls was compiled. Thick and thin blood smears stained with Giemsa were used to search for asexual forms and determination of parasite species. The CD4 + lymphocytes count, in each patient was performed on FACS Count. We did a distribution of HIV patients in three groups according to the $\mathrm{CD} 4+$ range: $350 \mathrm{CD} 4+/ \mathrm{mm}^{3}$. Demographic data were recorded. The statistical analysis was performed on the software Epi Info 3.5.

Results 511 PLHIV 33.3\% (95\% CI, 31 -35.8) and 1022 controls $66.7 \%$ (95\% CI, 62.2 to 69$)$ are included in the sample. Females were dominant with $60.7 \%$ (95\% CI, 58.2 to 63.2 ). The average age was $34.6 \pm 12.6$ years. The overall prevalence of parasitaemia $17.5 \%$ (268/1533, 95\% CI: 15.6 to 19.6). Parasitaemia in PLHIV $13.7 \%$ $(70 / 511)$ against $19.4 \%(198 / 1022)$ in controls $(\mathrm{P}<0.05)$. Parasitaemia in PLHIV related to CD4 + was $16.4 \%(11 / 67)$ in the 350 CD4 $+/ \mathrm{mm}^{3}(\mathrm{P}<0.05)$

Conclusions Cotrimoxazole appears to protect PLHIV against malaria but there was not much difference with the controls.

\section{P2.130 PENILE MONDOR'S DISEASE: AN UNDERDIAGNOSED AND UNDER-REPORTED BENIGN CONDITION}

doi:10.1136/sextrans-2013-051184.0394

\section{B Kumar. Silver Oaks Multi Specialty Hospital, Mohali-Punjab, India}

Bhushan Kumar, Abir Saraswat, BD Radotra, Tarun Narang Background Mondor's disease of the penis is usually a benign, self-limiting process of acute onset; associated with considerable psychological stress and sexual disharmony. Although it is not so uncommon, there have been few large studies.

Methods A retrospective review of 37 patients with penile Mondor's disease over a period of 2002-2010 was done. Detailed history and clinical findings were recorded. Histopathological examination and staining with CD31 and CD34 monoclonal antibodies was also done in 17 patients. Patients were followed up to see the response to treatment and recurrences. 